\title{
Review of: "Acute ischemic stroke triggers a cellular senescence-associated secretory phenotype"
}

\author{
Nathan d'adesky
}

Potential competing interests: The author(s) declared that no potential competing interests exist.

Cellular senescence describes an impairment in mitotic cell's ability to replicate and may be contributed to the exposure stressful stimuli to cells (aging, oxidative stress, DNA damage). Activation of senescent pathways have been shown to involve the expression of the tumor suppressors p16 and p21. Once senescent pathways become activated, cells express altered gene expression pathways involving distinct secretory profiles called the cellular senescence-associated secretory phenotype which may contribute to further spread of senescence to surrounding cells through cell signaling.

In this article, the authors report data that support a cellular senescence-associated secretory phenotype after a cerebral ischemic event in both mouse and human studies. To do so, transient middle cerebral artery occlusion is used in a mouse model to mimic stroke, and post-stroke brain tissue was collected for mRNA expression and immunohistochemistry. Human tissue was also used in a separate analysis using immunohistochemistry. Briefly, the authors main findings include a significant increase in p16, p21 Cxcl1 and Cxcr2 mRNA 72 hours after surgery in the infarct area vs contralateral. The study also demonstrates through immunohistochemistry p16 and p21 expression significantly increased in the infarct area vs. contralateral in both microglia and neurons.

The results of this study are interesting and support a cellular senescence secretory phenotype after ischemic stroke. Importantly, the study has important applications to possible further therapeutic options for post-stroke recovery which is currently limited to rehabilitative strategies. Although the study does provide evidence to support cellular senescence after stroke, there are a number of limitations to the study listed below:

- The inclusion of subjects exposed to middle cerebral artery occlusion required a reduction of less than $80 \%$ of baseline flow to be included. Author may consider using a more stringent requirement to prevent non-ischemic subjects in the study and to reduce type II error.

-This study lists the species, age, and sex of mice. However, the study only uses male mice, which excludes drawing any information about sex differences in cellular senescence. 
-The experiment only contains two human samples is without non-stroke controls. Test subjects are complicated by comorbidities (cirrhosis, sepsis, and aging) which confound the data, and significantly impact drawing any conclusions based on the human data.

-This study only uses young mice (12 weeks old) which does not correlate with the predominate population of stroke patients being middle aged/elderly. Older subjects may exhibit baseline senescent phenotype or may respond differently to stroke than younger subjects. Including aged mice in future experiments would supplement knowledge in this field.

-Experiments are in general underpowered. For gene expression and cell cycle markers in MCAO mice only used 3 mice for 30 min after surgery and 5 mice for 72 h after surgery. Immunohistochemistry only used 3 mice. More test subjects would allow for greater confidence is results, and less room for findings based on random error.

- There are no sham surgery mice, which would help distinguish effect of surgery itself on results.

-Ipsilateral penumbra area was not shown on immunohistochemistry studies. This area may be a more significant area which reflects cells more likely to survive after ischemia.

-No legend was given to show what is represented by 2 stars vs. 4 stars in figure 1 